\title{
Viewpoint \\ Estrogen regulation of mammary gland development and breast cancer: amphiregulin takes center stage
}

\author{
Heather L LaMarca and Jeffrey M Rosen
}

Department of Molecular and Cellular Biology, Baylor College of Medicine, Baylor Plaza, Houston, TX 77030, USA

Corresponding author: Jeffrey M Rosen, jrosen@bcm.edu

Published: 20 July 2007

This article is online at http://breast-cancer-research.com/content/9/4/304

(c) 2007 BioMed Central Ltd
Breast Cancer Research 2007, 9:304 (doi:10.1186/bcr1740)

\section{Amphiregulin as a paracrine mediator of estrogen-induced ductal morphogenesis}

Several candidate paracrine factors have been suggested to regulate hormone-induced proliferation and morphogenesis, including Wnt-4, receptor activator of NFKB ligand (RANKL), growth hormone and insulin growth factor II (reviewed in [3]). With regard to estrogen action, however, amphiregulin (AREG) appears to be the major paracrine mediator of ductal morphogenesis [4]. AREG is a member of the epidermal growth factor receptor (EGFR) family of ligands that binds exclusively to EGFR (ErbB1). Unlike the other EGFR ligands, AREG is upregulated in the mammary gland during ductal elongation, rendering this growth factor a likely mediator of epithelial proliferation. Furthermore, ductal outgrowth is severely impaired in AREG ${ }^{-/-}$mice, similar to the phenotype displayed by $E R \alpha$-null mammary glands. Brisken and colleagues recently demonstrated that AREG mRNA levels are increased in response to estrogen in the puberal mammary gland and that this increase is mediated by ER $\alpha$ [4]. Analysis of AREG-null mammary glands at various developmental stages showed that AREG is essential for ductal elongation as well as estrogen-induced proliferation and terminal end bud formation. Additionally, GFP-tagged AREG-null mammary tissue transplanted into the cleared fat pads of WT mice failed to grow. Elegant chimeric transplantation experiments were again utilized to investigate whether AREG elicited a paracrine response. Strikingly, grafting of mosaic epithelia containing both AREG-null and WT cells recapitulated the phenotype exhibited by ER $\alpha$ chimeric glands. AREG-null epithelial cells were able to proliferate only when WT cells resided nearby, supporting the notion that AREG is an important paracrine regulator of estrogen-induced proliferation [4].

$\mathrm{ADAM}=$ a disintegrin and metalloproteinase; $\mathrm{AREG}=$ amphiregulin; $\mathrm{EGFR}=$ epidermal growth factor receptor; ER = estrogen receptor; GFP = green fluorescent protein; HELU = hyperplastic enlarged lobular unit; TDLU = terminal duct lobular unit; TGF = transforming growth factor; WT = wild type. 
Notably, previous studies have shown that EGFR in the stroma, but not the epithelium, is essential for ductal morphogenesis $[5,6]$. Studies by Werb and coworkers revealed a potential mechanism by which epithelial-derived AREG activates EGFR on neighboring stromal cells [7]. They demonstrated that in culture, ADAM17 (a disintegrin and metalloproteinase 17) activates AREG, which is normally secreted as an inactive, transmembrane precursor [7]. Similar to the phenotype observed in AREG-null mammary glands, ductal outgrowth was inhibited in ADAM17\% epithelium, and exogenous AREG could rescue ADAM17\% transplants [7]. These data suggest a key role for ADAM17 in the paracrine interaction elicited by estrogen signaling.

While intriguing, these results raise several critical questions. First, is AREG necessary and sufficient to rescue ductal morphogenesis in ER $\alpha$-null mammary epithelium, or are other paracrine factors involved? These types of genetic rescue experiments are required to validate the models proposed by Brisken and Werb and colleagues. Second, how does AREG signal through the basement membrane and the myoepithelium to act on EGFR in the stroma? Likewise, what is the reciprocal paracrine signal that stimulates the epithelium? Stromal-derived fibroblast growth factor 7 has been suggested to activate epithelial fibroblast growth factor receptor $2 \mathrm{~b}$ during mammary branching [3], but genetic evidence for this mechanism has yet to be provided.

\section{Estrogen receptor- $\alpha$ and mammary stem cells}

What insights do these studies provide with respect to the potential role of estrogen-mediated proliferation in mammary stem cells? Although considerable progress has been made in the characterization of mammary stem cells, the mechanisms that regulate their self-renewal remain poorly understood. Previous studies involving the role of steroid hormones in stem/progenitor cell activity have reported conflicting results. It has been proposed that slow-dividing, labelretaining ER $\alpha$-positive cells comprise a stem/progenitor cell population that can directly respond to hormones $[8,9]$. In striking contrast, Brisken and colleagues' studies suggest that non-proliferating ER $\alpha$-positive cells exploit a paracrine mechanism to stimulate the proliferation of neighboring stem/ progenitor cells [2,4]. Shackleton et al. [10] demonstrated that a single, self-renewing LinCD29hiCD24+ ${ }^{+}$cell could repopulate a cleared mammary fat pad. Further analysis of this basal population revealed that these cells were exclusively ER $\alpha$-negative, but did express proliferating cell nuclear antigen and EGFR [11]. Recent studies by Smalley and coworkers [12] have also demonstrated that a subpopulation of ER $\alpha$-positive epithelial cells lacked in vivo stem cell activity. Limiting dilution transplantation revealed that $C D 24^{\text {lo }}$ ER $\alpha$-negative basal epithelial cells displayed the highest stem cell activity, while ER $\alpha$-positive luminal cells exhibited very little stem cell activity, as defined by mammary repopulating units [12].
Figure 1

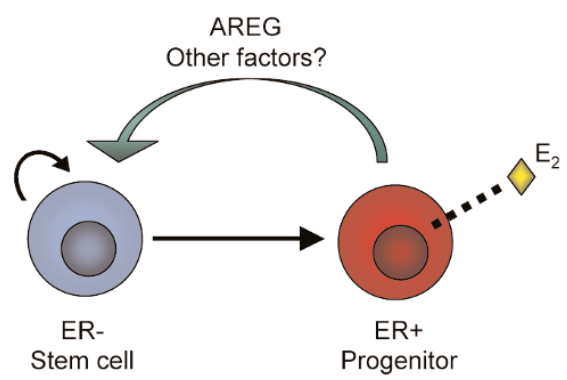

Mammary stem cells and the estrogen receptor (ER). ER-negative stem cells may asymmetrically divide to self-renew and to give rise to undifferentiated ER-positive progenitor cells. In response to estrogens, ER-positive progenitors secrete paracrine factors, such as amphiregulin (AREG) that can influence the proliferation and/or differentiation of ER-negative stem cells. $E_{2}$, estradiol.

These studies raise the following critical question: how can a single ER $\alpha$-negative stem cell give rise to a complete, functional ductal tree when transplanted into the cleared mammary fat pad? Wicha and colleagues [13] have proposed that ER $\alpha$-negative stem cells give rise to undifferentiated steroid receptor-positive cells, which proliferate in response to estrogen and secrete paracrine factors that regulate the proliferation and differentiation of adjacent ER-negative cells. In accordance with this model and the findings of Brisken and coworkers, we propose that an ER $\alpha$-negative basal stem cell may divide asymmetrically once to give rise to a luminal $E R \alpha$ positive progenitor cell. In response to estrogen stimulation, the resulting $E R \alpha$-positive cell may secrete paracrine factors, such as AREG or growth hormone, that can feedback on ER $\alpha$-negative stem cells (Figure 1). In addition, those paracrine factors may stimulate the proliferation and/or differentiation of adjacent $\mathrm{ER} \alpha$-negative and $\mathrm{ER} \alpha$-positive progenitor cells. Finally, epithelial-derived AREG, for example, may bind EGFR on more differentiated, neighboring stromal cells that induce other local growth factors, resulting in massive proliferation during ductal morphogenesis (see Figure 3 of [3]).

\section{Amphiregulin and breast cancer}

What implications do these studies of normal mammary ductal morphogenesis have for our understanding of human breast cancer? Specifically, how do quiescent ER $\alpha$-positive epithelial cells become proliferative in hormone-dependent breast cancers? Some insights have come from recent studies by Allred and coworkers [14], who used DNA microarrays to determine differences in gene expression between terminal duct lobular units (TDLUs) and hyperplastic enlarged lobular units (HELUs). HELUs result from abnormal enlargement of TDLUs and are the earliest histologically identifiable lesions with premalignant potential observed during the progression from normal ductal epithelium to hormone-dependent breast cancer. Interestingly, HELUs, 
which were previously shown to contain highly proliferative cells with elevated ER $\alpha$ expression, showed a ten-fold increase in AREG compared to normal TDLUs. This increase was accompanied by a 14-fold decrease in epidermal growth factor, while the expression of EGFR was unchanged. These results suggest an early switch in EGFR ligands that may be important for breast cancer initiation [14]. Whether this switch is mediated by estrogen remains to be determined.

AREG is overexpressed in most ER $\alpha$-positive primary breast tumors, and EGFR expression in breast cancer has been associated with poor prognosis and resistance to hormone therapy [15]. Using a three-dimensional culture model of human breast cancer progression, Bissell and colleagues [16] recently described an ADAM17-dependent autocrine loop that induced a malignant phenotype. Importantly, this phenotype was reversed by inhibiting ADAM17, which consequently halted signals exerted by AREG and transforming growth factor (TGF)- $\alpha$, another member of the EGFR ligand family. Bissell proposed that ADAM17 is a key protease involved in subverting the dependence on proliferative signals in breast cancer progression, and that targeting ADAM17 activity may provide an effective therapy for those breast cancers with deregulated EGFR signaling [16]. It should be noted, however, that the particular ligand that is important in EGFR-overexpressed breast cancers remains controversial. Other studies have suggested that TGF- $\alpha$, rather than AREG, plays a key role in breast cancer progression [17]. In support, Bissell and coworkers [16] showed a direct correlation between ADAM17 and TGF- $\alpha$ in a panel of human breast tumors, which was predictive of poor prognosis. Thus, it is clear that further investigation is required to elucidate the mechanistic role of AREG and/or TGF- $\alpha$ in hormone-dependent breast cancers.

\section{Conclusions}

Estrogen-mediated proliferation is fundamental to mammary gland development and breast tumorigenesis. Recent studies have established an important relationship between ER $\alpha$, AREG, proliferation and breast cancer. AREG is a key paracrine factor that not only mediates ductal elongation, but may also play an important role in mammary stem cell selfrenewal and differentiation. Elucidating the molecular mechanisms that regulate AREG signaling in the mammary gland, including the switch from estrogen-mediated paracrine events to an autocrine pathway, will be required for a complete understanding of the early events in breast cancer progression.

\section{Competing interests}

The authors declare that they have no competing interests.

\section{Acknowledgements}

Heather LaMarca is the recipient of an American Cancer Society Tricam Industries Postdoctoral Breast Cancer Fellowship (PF-06-25201-MGO).

\section{References}

1. Korach KS, Couse JF, Curtis SW, Washburn TF, Lindzey J, Kimbro KS, Eddy EM, Migliaccio S, Snedeker SM, Lubahn DB, et al.: Estrogen receptor gene disruption: molecular characterization and experimental and clinical phenotypes. Recent Prog Horm Res 1996, 51:159-186; discussion 186-158.

2. Mallepell S, Krust A, Chambon P, Brisken C: Paracrine signaling through the epithelial estrogen receptor alpha is required for proliferation and morphogenesis in the mammary gland. Proc Natl Acad Sci USA 2006, 103:2196-2201.

3. Sternlicht MD: Key stages in mammary gland development: the cues that regulate ductal branching morphogenesis. Breast Cancer Res 2006, 8:201.

4. Ciarloni L, Mallepell S, Brisken C: Amphiregulin is an essential mediator of estrogen receptor alpha function in mammary gland development. Proc Natl Acad Sci USA 2007, 104:54555460.

5. Sebastian J, Richards RG, Walker MP, Wiesen JF, Werb Z, Derynck R, Hom YK, Cunha GR, DiAugustine RP: Activation and function of the epidermal growth factor receptor and erbB-2 during mammary gland morphogenesis. Cell Growth Differ 1998, 9:777-785.

6. Wiesen JF, Young P, Werb Z, Cunha GR: Signaling through the stromal epidermal growth factor receptor is necessary for mammary ductal development. Development 1999, 126:335344.

7. Sternlicht MD, Sunnarborg SW, Kouros-Mehr H, Yu Y, Lee DC, Werb Z: Mammary ductal morphogenesis requires paracrine activation of stromal EGFR via ADAM17-dependent shedding of epithelial amphiregulin. Development 2005, 132:3923-3933.

8. Clarke RB, Anderson E, Howell A, Potten CS: Regulation of human breast epithelial stem cells. Cell Prolif 2003, 36(Suppl 1):45-58.

9. Zeps N, Dawkins HJ, Papadimitriou JM, Redmond SL, Walters MI: Detection of a population of long-lived cells in mammary epithelium of the mouse. Cell Tissue Res 1996, 286:525-536.

10. Shackleton M, Vaillant F, Simpson KJ, Stingl J, Smyth GK, AsselinLabat ML, Wu L, Lindeman GJ, Visvader JE: Generation of a functional mammary gland from a single stem cell. Nature 2006, 439:84-88.

11. Asselin-Labat ML, Shackleton M, Stingl J, Vaillant F, Forrest NC, Eaves CJ, Visvader JE, Lindeman GJ: Steroid hormone receptor status of mouse mammary stem cells. J Natl Cancer Inst 2006, 98:1011-1014.

12. Sleeman KE, Kendrick H, Robertson D, Isacke CM, Ashworth A, Smalley MJ: Dissociation of estrogen receptor expression and in vivo stem cell activity in the mammary gland. J Cell Biol 2007, 176:19-26.

13. Dontu G, El-Ashry D, Wicha MS: Breast cancer, stem/progenitor cells and the estrogen receptor. Trends Endocrinol Metab 2004, 15:193-197.

14. Lee S, Medina D, Tsimelzon A, Mohsin SK, Mao S, Wu Y, Allred DC: Alterations of gene expression in the development of early hyperplastic precursors of breast cancer. Amer J Path, 2007, 171:252-262.

15. Johnston SR: Clinical efforts to combine endocrine agents with targeted therapies against epidermal growth factor receptor/human epidermal growth factor receptor 2 and mammalian target of rapamycin in breast cancer. Clin Cancer Res 2006, 12:1061s-1068s.

16. Kenny PA, Bissell MJ: Targeting TACE-dependent EGFR ligand shedding in breast cancer. J Clin Invest 2007, 117:337-345.

17. Alwan HA, van Zoelen EJ, van Leeuwen JE: Ligand-induced lysosomal epidermal growth factor receptor (EGFR) degradation is preceded by proteasome-dependent EGFR de-ubiquitination. J Biol Chem 2003, 278:35781-35790. 